\title{
An idealised study for the long term evolution of crescentic bars
}

\author{
W.L. Chen, ${ }^{a}, *$ N. Dodd, ${ }^{a}$ M.C.H. Tiessen, ${ }^{b}$ D. Calvete ${ }^{c}$ \\ ${ }^{a}$ Faculty of Engineering, University of Nottingham, Nottingham NG7 2RD, UK. \\ ${ }^{\mathrm{b}}$ Deltares, Marine and Coastal Systems Unit, Department of Environmental \\ Hydrodynamics, Rotterdamseweg 185, 2629 HD Delft, The Netherlands. \\ ${ }^{\mathrm{c}}$ Department de Física Aplicada, Universitat Politècnica de Catalunya, Jordi \\ Girona 1-3, E-08034 Barcelona, Spain.
}

\begin{abstract}
An idealised study that identifies the mechanisms in the long term evolution of crescentic bar systems in nature is presented. Growth to finite amplitude (i.e., equilibration, sometimes referred to as saturation) and higher harmonic interaction are hypothesised to be the leading nonlinear effects in long-term evolution of these systems. These nonlinear effects are added to a linear stability model and used to predict crescentic bar development along a beach in Duck, North Carolina (USA) over a 2-month period. The equilibration prolongs the development of bed patterns, thus allowing the long term evolution. Higher harmonic interaction enables the amplitude to be transferred from longer to shorter lengthscales, which leads to the dominance of shorter lengthscales in latter post-storm stages, as observed at Duck. The comparison with observations indicates the importance of higher harmonic interaction in the development of nearshore crescentic bar systems in nature. Additionally, it is concluded that these nonlinear effects should be included in models simulating the development of different bed patterns, and that this points a way forward for long-term morphodynamical modelling in general.
\end{abstract}

Key words: Crescentic bed-patterns, linear stability analysis, field observations, long term evolution, nearshore morphology, higher harmonic interaction

* Corresponding author, wenlong. chen@nottingham.ac.uk 
Nearshore sea bed patterns are a common feature around the world and may provide some protection to beach and coastal areas (Hanley et al., 2014). As one of the most common nearshore sea bed patterns, crescentic bars are observed worldwide, see e.g. Van Enckevort et al. (2004). Such near shore sand bars can reduce wave momentum flux, or radiation stress, as the wave breaking on top of it. Furthermore, it can also provide sand to the beach if it migrates onshore (Ribas et al., 2015b). Because of their prevalence, their possible role in coastal protection, and the need to gain more understanding of nearshore coastal dynamics in general, it is important to study the evolution of these morphological features.

Increasingly, the genesis of such quasi-periodic patterns is thought to be due to morphological instability (see Ribas et al., 2015a). An often used method for describing the development of crescentic bed-forms in idealised scenarios is therefore linear stability analysis, see e.g. Deigaard et al. (1999); Falqués et al. (2000); Damgaard et al. (2002); Calvete et al. (2005); Van Leeuwen et al. (2006); Calvete et al. (2007). In this method, infinitesimally small perturbations are imposed on an equilibrium (basic) state. The interaction of flow and sea bed may give rise to a so called fastest growing mode, a bed-form with largest growth rate, which will dominate the sea bed pattern after a period of evolution. Linear stability analysis has proved to be useful in revealing the initialization and short term evolution of crescentic bars.

Following this approach, Tiessen et al. (2010) predicted the development of crescentic bed-patterns at Duck, North Carolina (USA), for a period of two months, starting from an along-shore constant bed. The forcing used was the measured wave and tidal data at the same field site. Although the predicted crescentic pattern lengthscales were similar to those observed, they tended to exhibit a much bigger fluctuation. Such significant discrepancy is found to be a combined result of missing nonlinear effects in the linear model and the effect of pre-existing bed patterns in the natural environment. This is because linear stability analysis is limited when pre-existing bed-forms are present, since an alongshore constant initial bathymetry is assumed at each instant. Another reason is that the exponentially growing bed form will violate the small amplitude assumption after some time, and nonlinear effects will dominate the evolution thenceforth. Therefore, a nonlinear analysis is necessary for reliable long-term prediction of crescentic bars (Dodd et al., 2003).

Using fully nonlinear numerical models, Tiessen et al. (2011) and Smit et al. (2012) included nonlinear effects and investigated the impact of pre-existing bed-patterns. Smit et al. (2012) showed that pre-existing bed-patterns 'with significant variability' do not adapt to changed hydrodynamic conditions, and 
dominate subsequent development. Moreover, such tendency holds for increasing wave energy. This suggests that, under certain circumstances, pre-existing modes are not affected by the present forcing conditions and that once a certain threshold of development is reached, only a reset-event, such as a storm, can remove pre-existing bed-forms and the corresponding dominant crescentic bed-pattern lengthscale.

On the other hand, Tiessen et al. (2011) showed that pre-existing modes can modify the subsequent development of different crescentic bar lengthscales. Pre-existing modes (patterns) of finite amplitude will persist if those same modes show significant linear growth (i.e., initial growth from an infinitesimally disturbed beach). On the contrary, pre-existing lengthscales that show only limited growth or even decay when developing from an infinitesimally disturbed beach, become overwhelmed by faster growing modes. However, the lengthscale of these pre-existing, slowly growing or decaying modes, and that of the newly-arising crescentic bed-form are linked. This is because the more rapid initial development of higher harmonics of the pre-existing lengthscale can excite a linearly unstable mode at a smaller wavelength, prior to decaying to insignificance.

The findings of Tiessen et al. (2011) and Smit et al. (2012) suggested a few important nonlinear effects in the long-term evolution of crescentic bars: higher harmonic interaction, persistence of bed-forms through weak storm and the importance of pre-existing bed-forms. Although the long term development of crescentic bars has been studied by many nonlinear numerical studies (e.g. Garnier et al., 2008; Castelle and Ruessink, 2011; Tiessen et al., 2011; Smit et al., 2012), all the existing nonlinear modelling studies are so far restricted to idealised simplified cases. Therefore, the existing knowledge of important nonlinear effects in the long-term evolution of crescentic bars lacks comparison with observations.

The goal of this study is therefore to identify physical mechanisms for longterm growth of crescentic bar systems by comparing with field observations.

To this end, we develop an idealised model that incorporates the processes suggested by Tiessen et al. (2011) and Smit et al. (2012) into the linear stability analysis. The occurrence of pre-existing modes is also accounted for in the model. This approach allows us to consider only those effects identified earlier, and, moreover, is time efficient and so can be applied over substantial durations. The model is used to predict the lengthscale of the crescentic bed-forms for a period of two months in 1998 at Duck (NC, USA). The model results are compared with field observation (Van Enckevort et al., 2004) over the same period.

The paper is organized as follows. In section 2 the model formulation is given, 
as well as how linear stability theory is used in the amplitude evolution model In section 3 the amplitude evolution model is presented, and an example test case used to illustrate its properties. Model results and a discussion are presented in section 4 and 5, respectively. Finally, a conclusion is given in section 6 .

\section{Model formulation: governing equations and linear stabiity anal- ysis}

The model geometry describes an unbounded, straight alongshore uniform open coast, with an example of cross-shore profile being shown in Fig. 1. Quasisteady flow conditions are assumed and the spatial coordinate system, $(x, y)$ in $\mathrm{m}$, is aligned with cross- and long-shore directions. The vertical direction is denoted by $z(\mathrm{~m})$, where $z=0$ refers to mean sea level with positive $z$ points upwards.

The model-framework is composed of the phase-averaged shallow water equations, in combination with a description of the bathymetric evolution, the wave phase and the wave energy density (see Calvete et al. (2005) for a more extensive description of this model).

The equations of the model are:

$$
\begin{aligned}
\frac{\partial D}{\partial t}+\frac{\partial D u_{j}}{\partial x_{j}} & =0 \\
\frac{\partial u_{i}}{\partial t}+u_{j} \frac{\partial u_{i}}{\partial x_{j}}=-g \frac{\partial z_{s}}{\partial x_{i}}-\frac{1}{\rho D} \frac{\partial}{\partial x_{j}}\left(S_{i j}^{\prime}-S_{i j}^{\prime \prime}\right)-\frac{\tau_{b i}}{\rho D} & \frac{\partial E}{\partial t}+\frac{\partial}{\partial x_{j}}\left(\left(u_{j}+c_{g_{j}}\right) E\right)+S_{i j}^{\prime} \frac{\partial u_{j}}{\partial x_{i}}=-\mathcal{D} \\
\frac{\partial \Phi}{\partial t}+\sigma+u_{j} \frac{\partial \Phi}{\partial x_{j}} & =0 \\
\frac{\partial z_{b}}{\partial t}+\frac{1}{1-p} \frac{\partial q_{j}}{\partial x_{j}} & =0
\end{aligned}
$$

where $i, j=1,2$, with summation being on $j ; x_{1,2}=(x, y)$ and $u_{1,2}=(u, v)$, where $u$ and $v\left(\mathrm{~ms}^{-1}\right)$ are the cross- and alongshore depth-averaged current respectively. $t(\mathrm{~s})$ represents time. $z_{s}(x, y, t)$ is the mean sea level, $z_{b}(x, y, t)$ is the mean bed level and $D$ is the total mean depth $\left(D=z_{s}-z_{b}\right) . E(x, y, t)(\mathrm{kg}$ $\mathrm{s}^{-2}$ ) is the wave energy density, which can be expressed in terms of the wave height $\left(E=\frac{1}{8} \rho g H_{r m s}^{2}\right) \cdot \tau_{b i}\left(\mathrm{~kg} \mathrm{~m}^{-1} \mathrm{~s}^{-2}\right)$ represents the bed shear stress; here the expression of Feddersen et al. (2000) is used. $g\left(\mathrm{~m} \mathrm{~s}^{-2}\right)$ is the gravitational 
acceleration, $\Phi$ (rad) is the wave phase and $\sigma(\mathrm{Hz})$ is the intrinsic frequency. The sediment flux $\left(q_{i}\right.$, in $\left.\mathrm{kg} \mathrm{s}^{-1}\right)$ is represented by the formula of Soulsby and Van Rijn (Soulsby, 1997). The bed porosity $p$ is 0.4 and the seawater density $(\rho)$ is $1024 \mathrm{~kg} \mathrm{~m}^{-3} \cdot S_{i j}^{\prime}\left(\mathrm{kg} \mathrm{s}^{-2}\right)$ is the radiation stress term and $S_{i j}^{\prime \prime}\left(\mathrm{kg} \mathrm{s}^{-2}\right)$ represents the Reynolds stresses (Calvete et al., 2005). $\mathcal{D}\left(\mathrm{kg} \mathrm{s}^{-3}\right)$ is the wave energy dissipation due to wave breaking described according to Church and Thornton (1993).

\subsection{Linear stability analysis}

In a linear stability analysis, the variables consist of an alongshore- and time invariant solution of (1)-(5), the basic state, denoted here with a zero subscript, and a small perturbation to that solution.

$$
\begin{aligned}
\left\{z_{s}, z_{b}, u_{1}, u_{2}, E, \Phi\right\}= & \left\{Z_{s 0}(x), Z_{b 0}(x), 0, V_{0}(x), E_{0}(x), \Phi_{0}(x, t)\right\} \\
& +\Psi(x) \exp (\omega t+\mathrm{i} k y) .
\end{aligned}
$$

The basic state corresponds to the wave conditions and water levels pertaining throughout the 2 months at Duck (see $\S 2.2$ ). It contains bed level $Z_{b 0}$, mean water level $Z_{s 0}$, alongshore current $V_{0}$, wave energy density $E_{0}$ and phase $\Phi_{0}$. The second term on the right hand side of (6) is the perturbation. The disturbances considered are alongshore-periodic with arbitrary wavelength $\lambda=2 \pi / k$, and (complex) frequency $\omega=\omega_{r}+\mathrm{i} \omega_{i}$. Thus the real part of the frequency $w_{r}$ represents the growth rate of the periodic pattern, while the imaginary part $\omega_{i}$ is related to the corresponding migration rate $\left(c_{m}=-\omega_{i} / k\right)$. A pattern with positive $w_{r}$ indicates a mode unbounded in time, i.e. a growing mode. The growthrate is determined by the combined effect of wave forcing and bathymetry, and has been studied by Calvete et al. (2005). Among all growing modes, the one with largest $\omega_{r}$ is defined as Fastest Growing Mode (FGM). For a chosen $k$, the evolution of the perturbation is solved as an eigenvalue problem for eigenvalue $\omega$ and eigenfunction $\Psi$.

\subsection{Basic state: field observation at Duck, 1998}

The basic state consists of forcing, an assumed equilibrium beach state, and a corresponding flow field. This forcing is the observed wave and tidal conditions recorded over a two month period in 1998, from August 20th (day 232) until October 22nd (day 294)(Van Enckevort et al., 2004). Wave data were recorded at about $8 \mathrm{~m}$ water depth, around $1000 \mathrm{~m}$ offshore, at three hour intervals. The same frequency was therefore used to obtain predictions from the model. Bathymetric evolution was only recorded at the beginning 


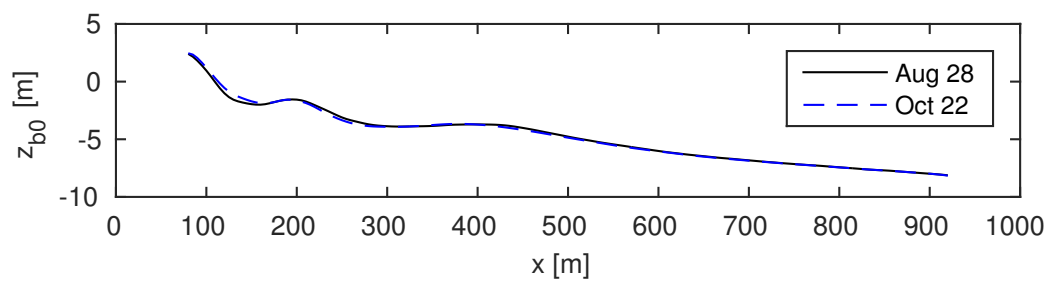

Fig. 1. Bed level profile resulting from alongshore averaging of the bathymetric surveys at the beginning and end of the two-month period.

and end of this 2-month period. So, the alongshore averaged bathymetric profile was determined every three hours by linear interpolation between the two alongshore-averaged profiles that were constructed from the full bathymetric surveys at the beginning and end of this period. In Fig. 1 we can see these two initial and final profiles.

Note that the tidal variation $\left(M_{2}\right)$ was included in the analysis by shifting the bathymetry vertically. The reproduced wave conditions and water depth are shown in Fig. 2. It can be seen that there are three times at which wave heights are increased for short durations (at about days 237, 263 and 272). We refer to these as storms 1, 2 and 3 respectively. Wave directions switch between northerly and southerly (with respect to the local coast), and so are likely to generate longshore currents in opposite directions at various times; some normally incident waves can also be seen. Periods are mostly confined within 5 and $15 \mathrm{~s}$. The tidal range is about $1 \mathrm{~m}$.

At each time interval, the observed wave data were applied on the offshore boundary of the linear stability model with updated bathymetric cross shore profile, to obtain predictions from the model. This will be further explained in $\S 2.4$.

\subsection{Growth rate curve}

As mentioned in $\S 2.1, k$ is arbitrary. So, we calculate the growth rate of all realistic morphodynamic lengthscales: $0.001<k<0.1\left[\mathrm{rad} \mathrm{m}^{-1}\right]$, for increments $\Delta k=0.001 \mathrm{rad} \mathrm{m}^{-1}$; corresponding $\lambda$ values are approximately $\{6.3 \mathrm{~km}, 3.1 \mathrm{~km}, 2.1 \mathrm{~km}, 1.6 \mathrm{~km}, 1.3 \mathrm{~km} \ldots 65.4 \mathrm{~m}, 64.8 \mathrm{~m}, 64.1 \mathrm{~m}, 63.5 \mathrm{~m}, 62.8 \mathrm{~m}\}$, for each set of forcing conditions (every three hours). It is assumed that the predictions made for each set of forcing conditions are valid for the three hour period until a new set of conditions becomes available. We thus require an entire growth rate curve for this region of $k$ space for each three-hour prediction. This allows us to identify a unique growth rate for each $k$, in order to determine the amplitude development of each lengthscale.

The identification of an entire growth rate curve corresponding to physical 

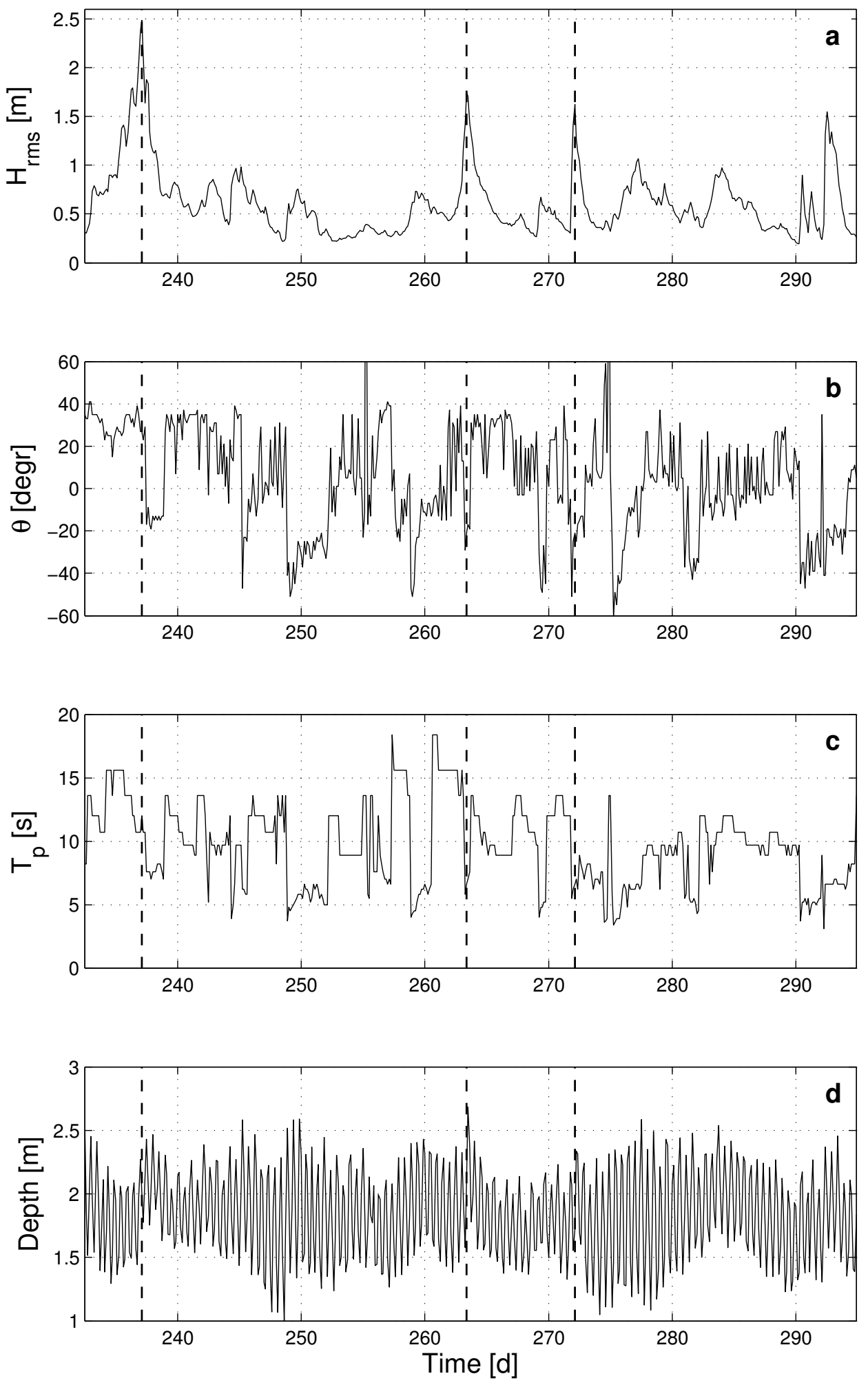

Fig. 2. Forcing conditions used in the linear stability model, as they were measured during observations period. (a) Wave height; (b) Wave angle relative to the coast $\left(0^{\circ}\right.$ is perpendicular to the coast, negative is from north to north-west and positive from west to south-west); (c) Wave peak period; (d) Water depth above the onshore bar. This water depth changes as a result of tides, surges and the bathymetric evolution. The vertical dashed lines represent storm events. 

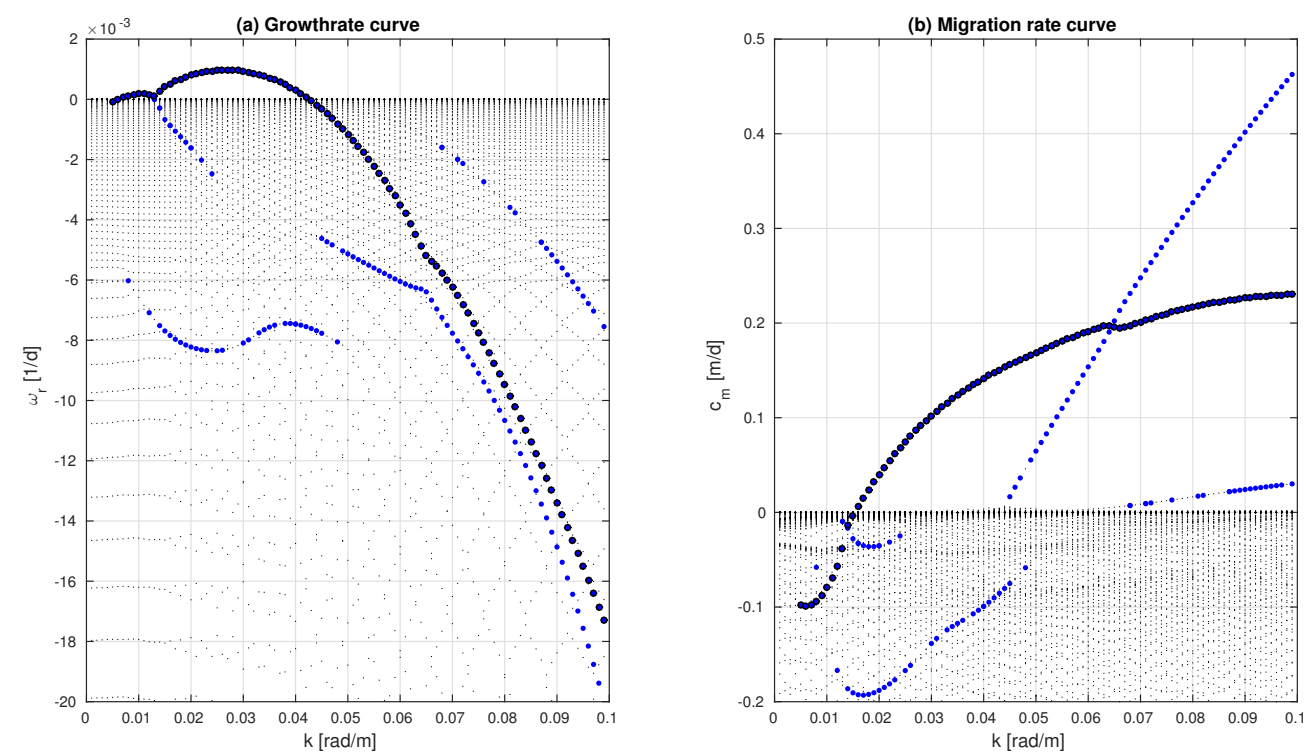

Fig. 3. (a) Growth rate $\left(\omega_{r}\right)$ curve; (b) Migration rate $\left(c_{m}\right)$ curve. Shown are the distribution for all $k$-values of the solutions of the system of equations, with small black dots for all solutions from Morfo60, blue dots for all physical modes and black encircled blue dots for selected physical mode.

modes is complicated due to the presence of spurious solutions to the equations. For each lengthscale, the number of possible solutions calculated equals the number $(n)$ of computational cross-shore nodes, with most of these results only describing physically meaningless spurious (i.e. non-physical) solutions to the system. These spurious solutions generally display negative or near-zero growth rates and, therefore, obscure in particular the negative part of the physical growth rate curve.

For all modes we must be sure that we have correctly identified physical modes. These physical modes are identified by testing the convergence of eigenvalues and eigenfunctions as $n$ increases. Spurious modes do not exhibit convergence and therefore are discarded. Runs were carried out with $300(n=300)$ and 450 nodes $(n=450)$. According to Calvete et al. (2005), 300 cross-shore nodes is sufficient to achieve convergence. Our tests lead to agreement with this condition.

This is done for all wavenumbers, resulting in multiple physical growth rate curves. An example of these curves is shown in Fig. 3. Among these physical growth rate curves, the one containing the highest growth rate for the region of $k$ space being examined is selected. This growth rate curve is considered to be the one that governs evolution of bed-forms for the 3 hours during which those forcing conditions pertain. Note, however (Fig. 3), that other physical curves do exist; we ignore these. 

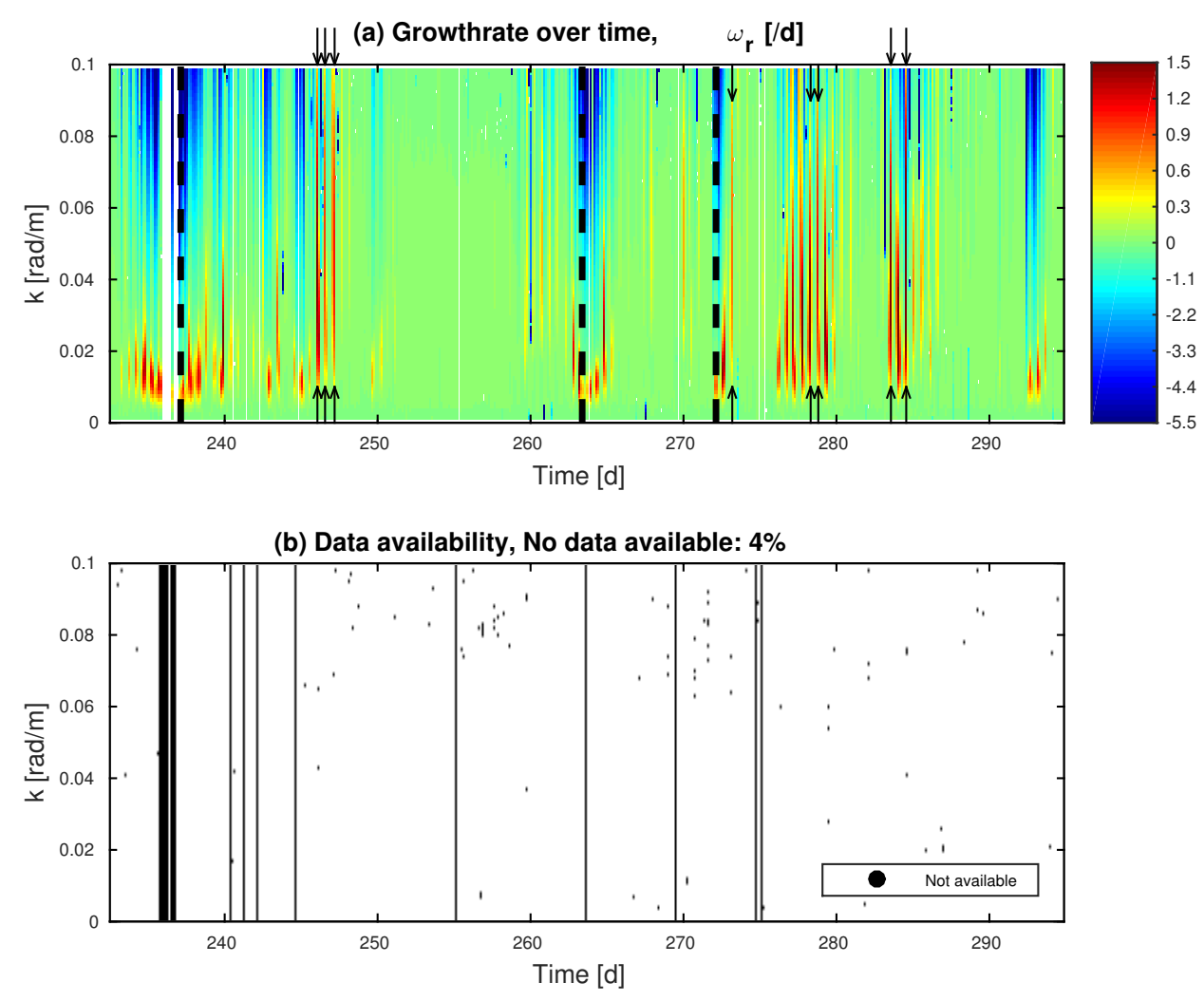

Fig. 4. (a) The growth rate curve at each time step as derived by selecting the physical growth rate curve as described in $\S 2.3$ and 2.4. Blue indicates negative growth rate and red positive growth rate, and the black dashed line indicates the time of the peak of a storm. Black arrows indicate example of circumstances which all values of $\mathrm{k}$ have positive growth rate. (b) Durations where no growth rate curve could be determined (black dots and bars denote the situation where no data is available).

\subsection{Growth rate over time}

Every three hours, a separate prediction of the linear growth rate curve is created based on the new hydrodynamic forcing conditions and bathymetry. The variability of this growth rate curve over time is significant (see Fig. 4(a)). Calmer conditions (as occur from day 255 to 259, for instance) generally result in very small growth rates, whereas bigger wave heights (as can be observed after day 237 in Fig. 2) result in both rapidly growing and decaying modes. The effect of the tidal variation $\left(M_{2}\right)$ can clearly be seen in the periodically varying growth rate. In low tide conditions, there are a few circumstances (highlighted by dark arrows in Fig. 4a) that show positive growth rates for a broad band of $\mathrm{k}$ (lengthscales).

The identification of the physical growth rates for each $k$-value has not been successful for all cases, as can be seen in Fig. 4(a, b). There are two situations when no physical growth rate could be obtained. Sometimes, the growth rate 
selected by the proposed method greatly deviates from neighbouring (in $k$ space) growth rates. In these circumstances we deem that result non-physical, and to avoid seemingly unrealistic results, we set $\omega_{r}=0$, see black dots in Fig. 4(b). Additionally, convergence is typically not achieved under more extreme storm conditions. When this occurred, it was again assumed that all lengthscales would show neither growth nor decay $\left(\omega_{r}=0\right)$, see vertical black bars in Fig. 4(b). For most of the cases, however, a growth rate is available. As shown in Fig. 4(b), the percentage of lengthscales that lack a physical growth and migration rate over time is about $4 \%$.

\section{Model formulation: amplitude development}

The bed-pattern lengthscale with the highest amplitude at any instant is deemed dominant and most likely to be observed in the field. Tiessen et al. (2010) took this lengthscale to be that corresponding to the $F G M$ at different times. Here we identify amplitude development for all lengthscales and derive the dominance of one lengthscale based on competition between these amplitudes, each of which is influenced by, but not solely dependent on, the linear growth rate.

A systematic approach to doing this is a weakly nonlinear perturbation expansion (see e.g. Schielen et al., 1993). This approach results in a rapidly increasing number of different harmonics of $k$. Motivated by Tiessen et al. (2011) we limit our investigation to linear growth, self-limitation of that growth (i.e., equilibration, or saturation), and the generation of the first harmonic. This approach is in keeping with that of Knaapen and Hulscher (2001), who used data-assimilation techniques to derive coefficients of an ampltiude evolution equation that would result from a weakly nonlinear analysis. We thus hypothesise that the two most important nonlinear effects in the long-term development of crescentic bars are: i) equilibration of growing modes for all $k$ values; and ii) generation of higher harmonics by growing modes, which therefore allow energy to be transferred to smaller wavelengths. This generation is depicted schematically in Table. 1 . The $O\left(\epsilon^{0}\right)$ term is our basic state, which remains unchanged. We consider the linearly growing (fundamental) mode (at $O\left(\epsilon^{1}\right)$ ), and the first harmonic $\left(O\left(\epsilon^{2}\right)\right)$ that it generates by self-interaction. As noted, we exclude alterations to the mean bed (basic state). Being a mean component this will not affect lengthscale evolution. However, interaction of the mean term with the fundamental mode (that of the linear instability) will give rise to an equilibration (saturation) term at $O\left(\epsilon^{3}\right)$; this is included. Second and higher harmonics are excluded. Note also that we assume this model to pertain for all $k$ values.

We choose the generic amplitude equation that can result from a weakly 


\begin{tabular}{l|l|l|l|l|l|l|l} 
& $-3 k$ & $-2 k$ & $-k$ & 0 & $k$ & $2 k$ & $3 k$ \\
\hline$O\left(\epsilon^{0}\right)$ & & & & $\checkmark$ & & & \\
$O\left(\epsilon^{1}\right)$ & & & $\checkmark$ & & $\checkmark$ & & \\
$O\left(\epsilon^{2}\right)$ & & $\checkmark$ & & $\mathrm{x}$ & & $\checkmark$ & \\
$O\left(\epsilon^{3}\right)$ & $\mathrm{x}$ & & $\checkmark$ & & $\checkmark$ & & $\mathrm{x}$ \\
\hline \hline
\end{tabular}

Table 1

Schematic depiction of the harmonics included in the amplitude evolution model; a $\checkmark(\mathrm{x})$ indicates inclusion (exclusion). $\epsilon$ represents the (small) amplitude of the bed pattern.

nonlinear analysis, which embodies the energy transfers described above (see Drazin and Reid, 1981). This is:

$$
\frac{d A_{k}}{d t}=\omega_{r k}\left(t_{n}\right) A_{k}-l_{k}\left(t_{n}\right) A_{k}^{3}+m_{k / 2}\left(t_{n}\right) A_{k / 2}^{2} .
$$

Note that $A_{k}(t)$ is our bed-form (mode) amplitude hereafter, where the $k$ subscript refers to the lengthscale to which this amplitude pertains (also for $\left.\omega_{r k}\right)$. The other coefficients in (7) are:

$$
l_{k}\left(t_{n}\right)=\left|\omega_{r k}\left(t_{n}\right)\right|, \quad m_{k / 2}=\alpha\left(1-A_{k}^{10}\right),
$$

where $\alpha$ is a constant. The first term on the right represents the linear growth (or decay). The amplitude $\left(A_{k}(t)\right)$ is therefore an initially exponentially growing (or decaying) quantity, assuming a small enough initial amplitude, with growth rate $\omega_{r k}\left(t_{n}\right) . A_{k}(t=0)=A_{\min }=0.1$ is the same for all lengthscales; this is also the minimum amplitude. During storm events, all pre-existing bedforms are expected to be erased. This is simulated by resetting the amplitudes of all lengthscales to $A_{\min }$. The maximum amplitude $A_{\max }=1$; as amplitudes approach this value it is assumed that nonlinear effects will become dominant, and so further linear development is assumed to cease as this limit is approached. The values of $A_{\min }$ and $A_{\max }$ do not convey any intrinsic meaning themselves, except that choosing $A_{\max }=1$ is consistent with the weakly nonlinear nature of the expansion (i.e. all powers of $A_{k}<1$ ) and can be done without loss of generality. The value of $A_{\min }$ therefore is arbitrary, except that a ten-fold growth seems to represent roughly the duration it takes for a crescentic bathymetry to reach a new stable situation after a storm.

This assumption regarding $A_{\max }$ motivates the choice for $l_{k}=\left|\omega_{r k}\left(t_{n}\right)\right|$, the coefficient of the second term on the right. This ensures the desired long-term behaviour. This $O\left(\epsilon^{3}\right)$ term represents the equilibration, and the amplitude equation including just the first two terms on the right is the Stuart-Landau equation (Drazin and Reid, 1981). which emerges in studies in fluid dynamics, and represents the effects of equilibration (growth to finite amplitude) only. The final term in (7) allows energy transfer to $A_{k}$ from lengthscales twice 
those of the lengthscale $\lambda=\frac{2 \pi}{k}$. The energy transfer factor, $\alpha=0.3$, was estimated based on the amplitude development rates of higher harmonic modes as observed by Tiessen et al. (2011), see Fig. 8 in Tiessen et al. (2011).

In $\S 5.4$ we examine the sensitivity of the simulations to changes in $\alpha$. The dependence of $m_{k / 2}$ on $A_{k}$ is included here to ensure that all modes can only achieve the same maximum amplitude, so that this term, if operational, accelerates growth only, and becomes inoperational as $\left|A_{k}\right| \rightarrow 1$. This dependence is the only part of (7) that would not result from a weakly nonlinear analysis.

\subsection{Numerical experiment on synthetic data}

Before applying (7) to the data-set for Duck, we first illustrate the effect of the various terms on the right of (7) by means of an idealised but (synthetic) representative example. This example notionally corresponds to two different forcing conditions consecutively applied for 12.5 days each. In Fig. 5 (a) and (b) we show the (time-invariant synthetic) growth rate curves corresponding to these two notional sets of forcing conditions. In Fig. 5 (c), (d) and (e) this results in the development of different crescentic bed-patterns with regards to lengthscale $\lambda$ (or $k$ ) and amplitude $\left(A_{k}\right)$, for three scenarios: Fig 5 (c) linear evolution (first term on the right of (7) only); Fig 5 (d) equilibration (first two terms on the right of (7) only); and Fig 5 (e) full model, i.e., linear evolution, equilibration and higher harmonic generation (all terms on the right of (7)).

In the early stages of linear evolution (Fig. 5(c)) there is rapid development of the lengthscale $\lambda_{1}=700 \mathrm{~m}$. This is the lengthscale of the $F G M$ for the first forcing condition (denoted here $F G M_{1}$, green line, see caption). After the first forcing conditions (Fig. 5(a)) have been applied for 12.5 days, the second set of forcing conditions (Fig. 5(b)) results in a decay of $F G M_{1}$, which remains dominant until the $F G M$ of the new conditions $\left(F G M_{2}\right.$, blue line) surpasses it. During day 23, $A_{k_{2}}$ exceeds $A_{\max }$, so further development is terminated. Note also the growth of lengthscale $\lambda_{1}^{\prime}=785 \mathrm{~m}\left(k_{1}^{\prime}\right)$ in the first 12.5 days: see Fig. 5 (a) and (c). This corresponds to that of the mode $F G M_{1}^{\prime}$ with growth rate almost as large as that of $F G M_{1}$. This mode grows and decays much like $F G M_{1}$.

For the equilibration case (Fig. 5(d)) bathymetric evolution is self limiting. As the amplitudes increase, again, centred around $k_{1}$ for the first 12.5 days, the rate of increase decreases, especially toward the end of this period. The subsequent transition from the first to the second forcing conditions (growth centred on $k_{1}$ to growth centred on $k_{2}$ ) leads to similar behaviour. However, now the amplitude development levels off when the amplitude approaches 1.

For the full model (Fig. 5(e)) we see qualitatively different behaviour. A small 

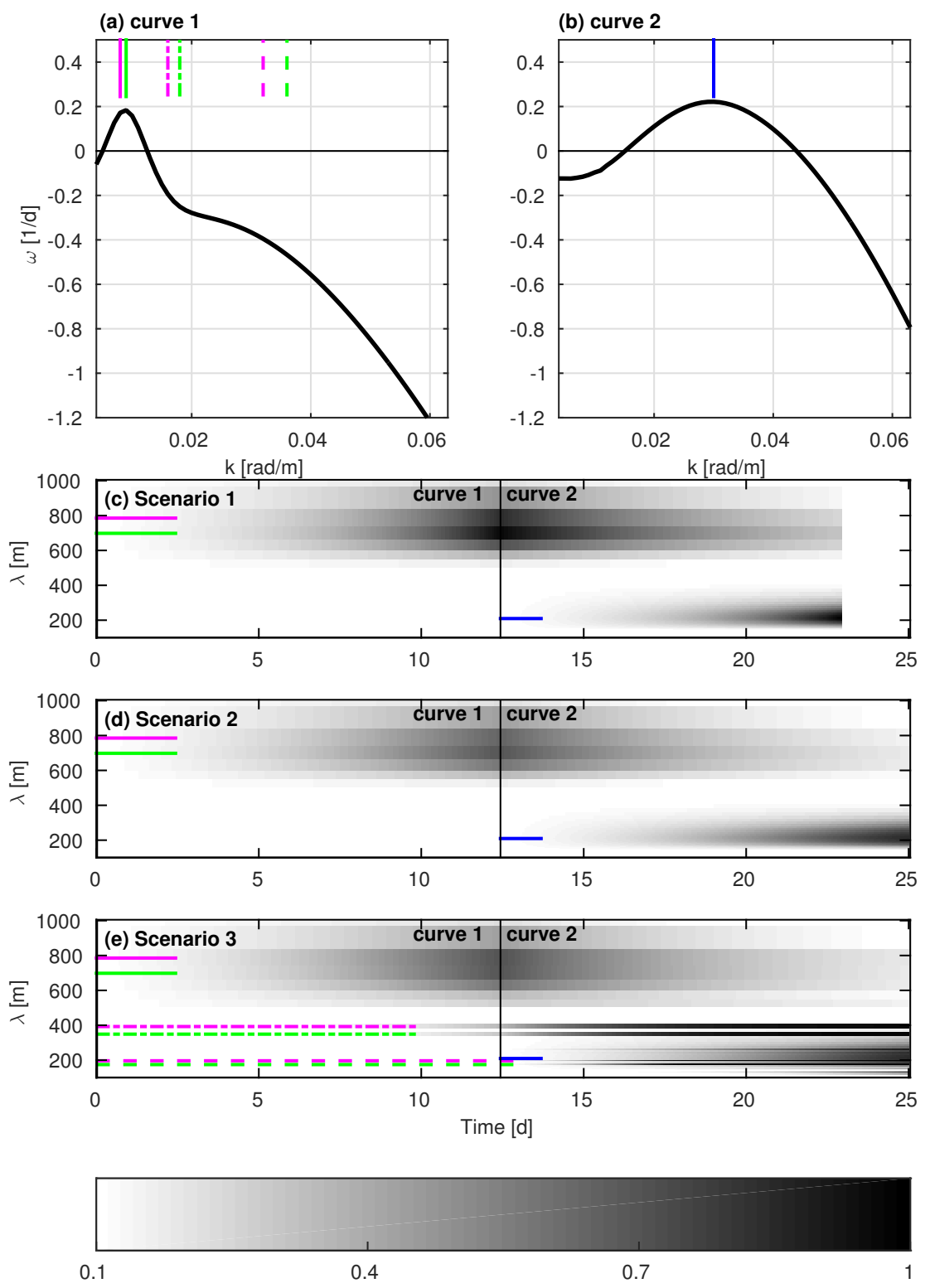

Fig. 5. Example of the three different cases: $(a, b)$ Two different growth rate curves applied consecutively for 12.5 days; (c) linear evolution only; (d) equilibrated solution; (e) full model. Light (dark) shading indicates low (high) amplitude. Coloured lines indicate the position in $k$ (in $\mathrm{rad} \mathrm{m}^{-1}$ ) space (a,b) or $\lambda$ (in m) space (c-e) of modes that exhibit significant growth in one or more cases. Solid lines: modes that only grow linearly. Green: $F G M_{1}$ (FGM corresponding to growth rate curve from the first forcing conditions, at $k=k_{1}=0.009 \mathrm{rad} \mathrm{m}^{-1}$ ); Magenta: $F G M_{1}^{\prime}$ (mode adjacent to $F G M_{1}$, for which $\omega_{r}$ is only slightly smaller than that for $F G M_{1}$ under first forcing conditions, $k=k_{1}^{\prime}=k_{1}-\Delta k=0.008 \mathrm{rad} \mathrm{m}^{-1}$ ); Blue: $F G M_{2}$ ( $F G M$ corresponding to the growth rate curve from second forcing conditions, at $\left.k=k_{2}=0.03 \mathrm{rad} \mathrm{m}^{-1}\right)$. Dash-dotted lines: Green: higher harmonic of $F G M_{1}\left(2 k_{1}\right)$; Magenta: higher harmonic of $F G M_{1}^{\prime}\left(2 k_{1}^{\prime}\right)$. Dashed lines: further higher harmonics $\left(4 k_{1}, 4 k_{1}^{\prime}\right)$ of $F G M_{1}$ and $F G M_{1}^{\prime}$. The lengths of the lines is for illustrative purpose only. 
but significant amount of energy is fed into $2 k_{1}$ and $2 k_{1}^{\prime}$ during the first 12.5 days, by higher harmonic generation. Under the second set of forcing conditions these wavelengths correspond to linearly growing modes, and so these continue to evolve during the latter 12.5 days. Additionally, $4 k_{1}$ and $4 k_{1}^{\prime}$ are similarly excited, and these modes lie close to $k_{2}$, so that even though they initially possess only limited amplitudes they ultimately grow rapidly. The result is a broader range of lengthscales (modes) containing significant amplitudes.

\section{Results}

\subsection{The evolution of crescentic bars}

The model predictions representing the two months of field observations at Duck (NC) for the three cases are shown in Fig. 6, where the amplitude development for all examined lengthscales is shown over time. We show the equivalent three cases to illustrate the effects of the inclusion of these physical mechanisms on predictions. For the predictions made solely by linear growth rates (Fig. 6(a)), the amplitude development is terminated when the fastest growing lengthscale reaches $A_{\max }$ (about day 246, after storm 1). In the field, the crescentic bars are likely to be removed during a storm (Van Enckevort et al., 2004). We thus assume that all pre-existing bed pattern are erased in a storm (shown as dashed lines), and predictions resume immediately after a storm. This eradication of pre-existing bed-forms during a storm is also applied for the other cases. During the subsequent bed evolution, the development of crescentic bars starts again from $A_{m i n}$.

The rate of development after the first and third storms is similar, which can be seen in the emergence of significant amplitudes at post-storm times. Although after storm 3, the significant amplitude emerges at a later post-storm time than that after storm 1. This development is larger than that after the second storm. The growth rate curve (Fig. 4(a)) shows why this difference happens. The only large growth rates after the second storm occur immediately after it, i.e., in a short period as the wave height is subsiding from its peak. In contrast, both the first and third post-storm periods exhibit significant durations when growth rates are significant (see the regions with 'red' growth rates in Fig. 4(a)). These durations roughly correspond to times when $H_{r m s}>0.5 m$ (see Fig. 2(a)). After storm 1, such duration of positive growth rate comes right after the storm, whereas after storm 3, such duration comes after a quiet period of roughly 4 days. This explains significant amplitudes emerge at a later poststorm time after storm 3 than after storm 1. Furthermore, the time interval between second and third storms is shorter than that between first and second storms, thus allowing less time for development of these bed-forms. 


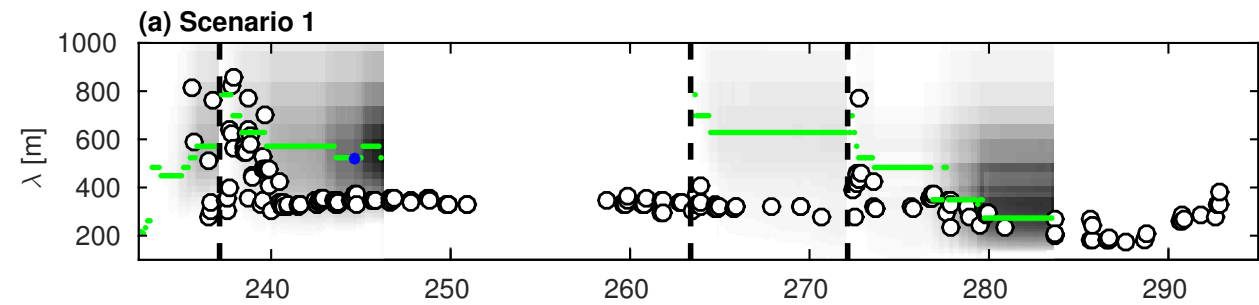

(b) Scenario 2

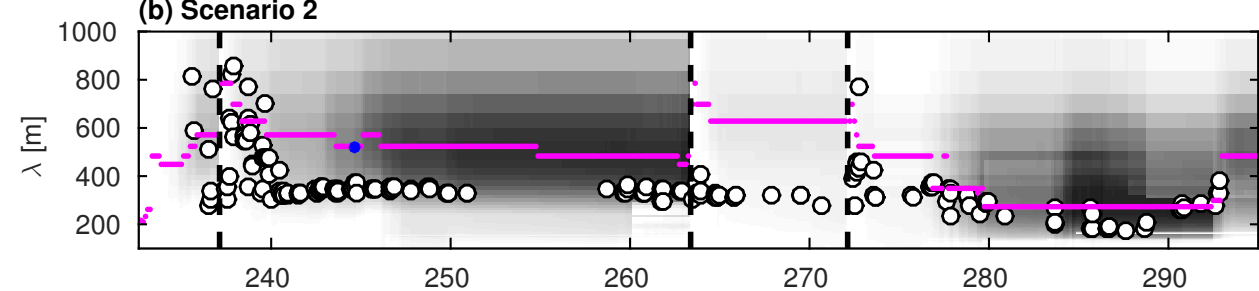

(c) Scenario 3
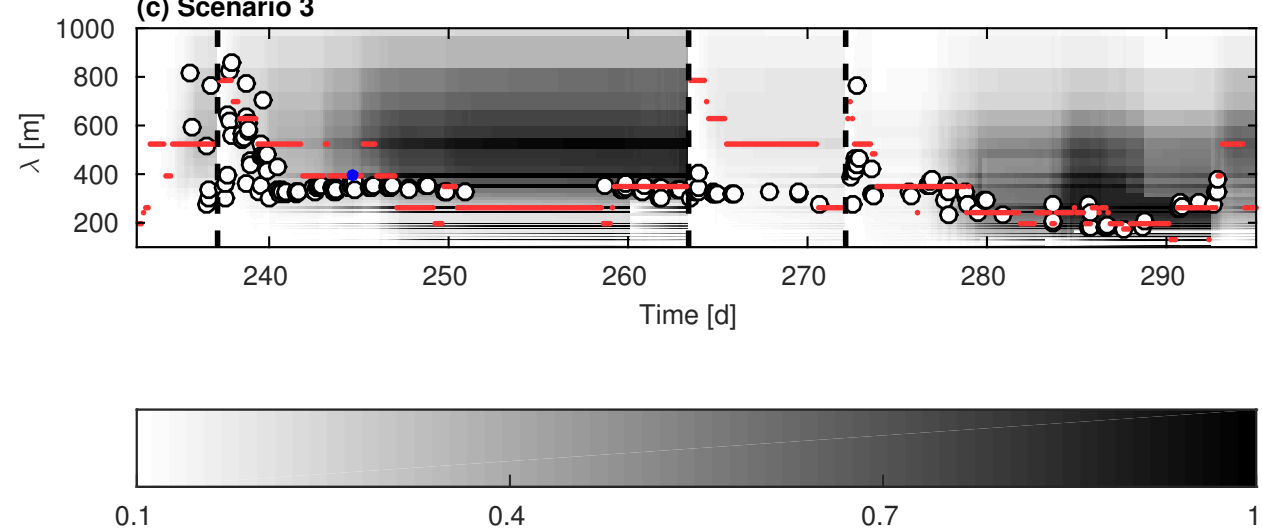

Fig. 6. Amplitude development for the three cases compared to the observed lengthscales (large white circles) (Van Enckevort et al., 2004), where coloured dots denote the predicted dominant lengthscale. (a) linear evolution; (b) equilibration; (c) full model.

For the equilibration case, development rates are reduced by the equilibration term during the latter post-storm stages. As a result, more gradual growth is seen latterly, but qualitatively behaviour is the same, except that the whole time period can now be accommodated.

In the case of higher harmonic interaction (full model), the simulation shows a significant amplitude transfer occurring from longer lengthscales to shorter lengthscales. This gives rise to a wider range of developing modes than is the case when only the linear evolution or equilibration are considered.

To better illustrate the model results, we reconstruct the sea bed patterns of dominant lengthscales with eigenfunctions calculated by Morfo60. An example is given in Fig. 7, showing the structure of the perturbations of dominant length scale. A crescentic bar shaped perturbation is observed on top of the alongshore bar (located at $88 \mathrm{~m}$ away from shoreline). With the inclusion of equilibration, the amplitude of the perturbation is smaller. In the full model 


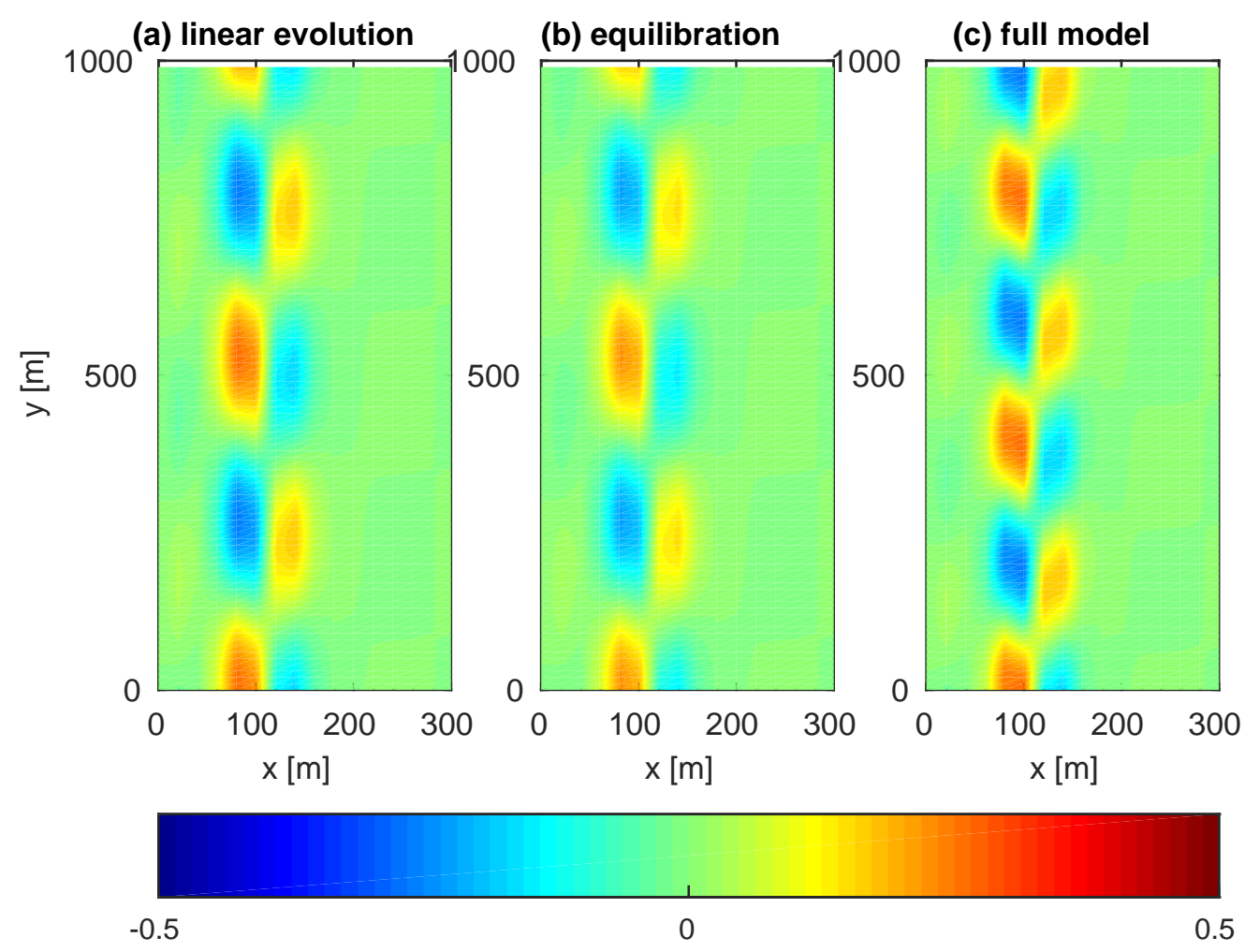

Fig. 7. Sea bed pattern of dominant length scales on day 245 (denoted by large blue dots in Fig. 6), for (a) linear evolution, with $\lambda=523.6 \mathrm{~m}$; (b) equilibration, with $\lambda=523.6 \mathrm{~m}$; (c) full model, with $\lambda=392.7 \mathrm{~m}$. Color indicates perturbation, with red and blue for positive and negative perturbations, respectively.

case, the perturbation shows a smaller lengthscale $(\lambda=392.7 \mathrm{~m})$ and is the higher harmonic mode of $\lambda=785.4 \mathrm{~m}$, which also exhibits significant amplitude in linear evolution case (see Fig. 6).

A comparison of the predicted and observed lengthscale evolution is also shown in Fig. 6. The predicted dominant lengthscale (that of the biggest amplitude at each time $t=t_{n}$ ) is shown as a coloured dot, and the observed lengthscales are shown as larger white dots. Note that the observation data is not available in between day 251 and day 259 (see the blank space of white circles in Fig. 6). In between storms, amplitude development based on linear evolution and equilibration generally over-predict the dominant lengthscale. Higher harmonic interaction (full model) results in a more rapid development of shorter lengthscales which is more in line with field observations (Fig. 6(c)). In some aspect, the full model reproduced the observed stabilisation of the bed-form lengthscales after storm 1, as the predicted lengthscale fluctuated in a narrow band of observed lengthscale. These fluctuations in predicted lengthscale, as can be seen from the amplitudes in Fig. 6(c), are due to relatively small amplitude differences between a number of co-existent modes. 


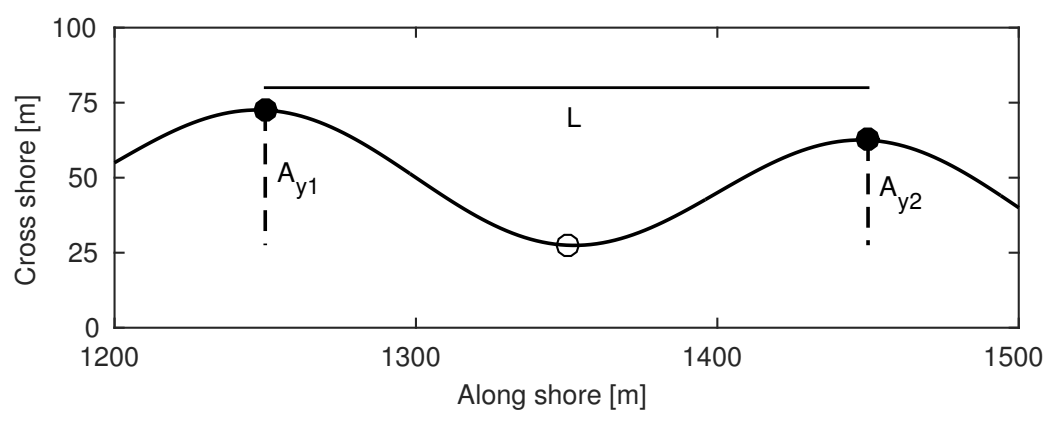

Fig. 8. A single crescent from the crescentic bar with length $L$ and horizontal amplitude $\bar{A}_{y}=0.5 \times\left(A_{y 1}+A_{y 2}\right) / 2$. The horn of the crescent is labelled with filled circles, whereas the bay is labelled with an open circle.

\subsection{Amplitude evolution}

Due to the lack of observational data of the vertical amplitude of crescentic bars, a straight comparison of the amplitude of the predicted dominant lengthscales with field observation is not possible. However, in Van Enckevort et al. (2004), the horizontal amplitude $\left(\bar{A}_{y}\right)$ of the crescentic bar at Duck is recorded. This amplitude was calculated as half the average cross-shore distance between the bay and the two horns (Figure 8). We hypothesize that the vertical amplitudes of crescentic bars is proportional to $\bar{A}_{y}$. In figure 9 , the predicted amplitude of the dominant lengthscale (solid black curve) is compared with the observed $\bar{A}_{y}$ (dash-dotted blue curve). In the full model evolution, amplitude growth and equilibration after storm 1 is consistent with that observed. After storm 3 the model produces more rapid growth to a higher amplitude than that observed, but, nonetheless, qualitatively similar behaviour. Again, the effect of the higher harmonic interactions may be observed by comparing figure 9 (b) and (c). The differences are small, but remember that the simulated amplitudes are those of the dominant lengthscale, and these are in general over predicted by the equilibration model. A substantial difference between the observation and simulation is found after storm 2. In a short period after storm 2, the observed amplitude recovers to the amplitude before the storm, whereas very limited amplitude development is observed in our model result. This, as also noted by Tiessen et al. (2010), points to the persistence of bed-forms through the second storm. This will be further discussed in $\S 5.2$. 

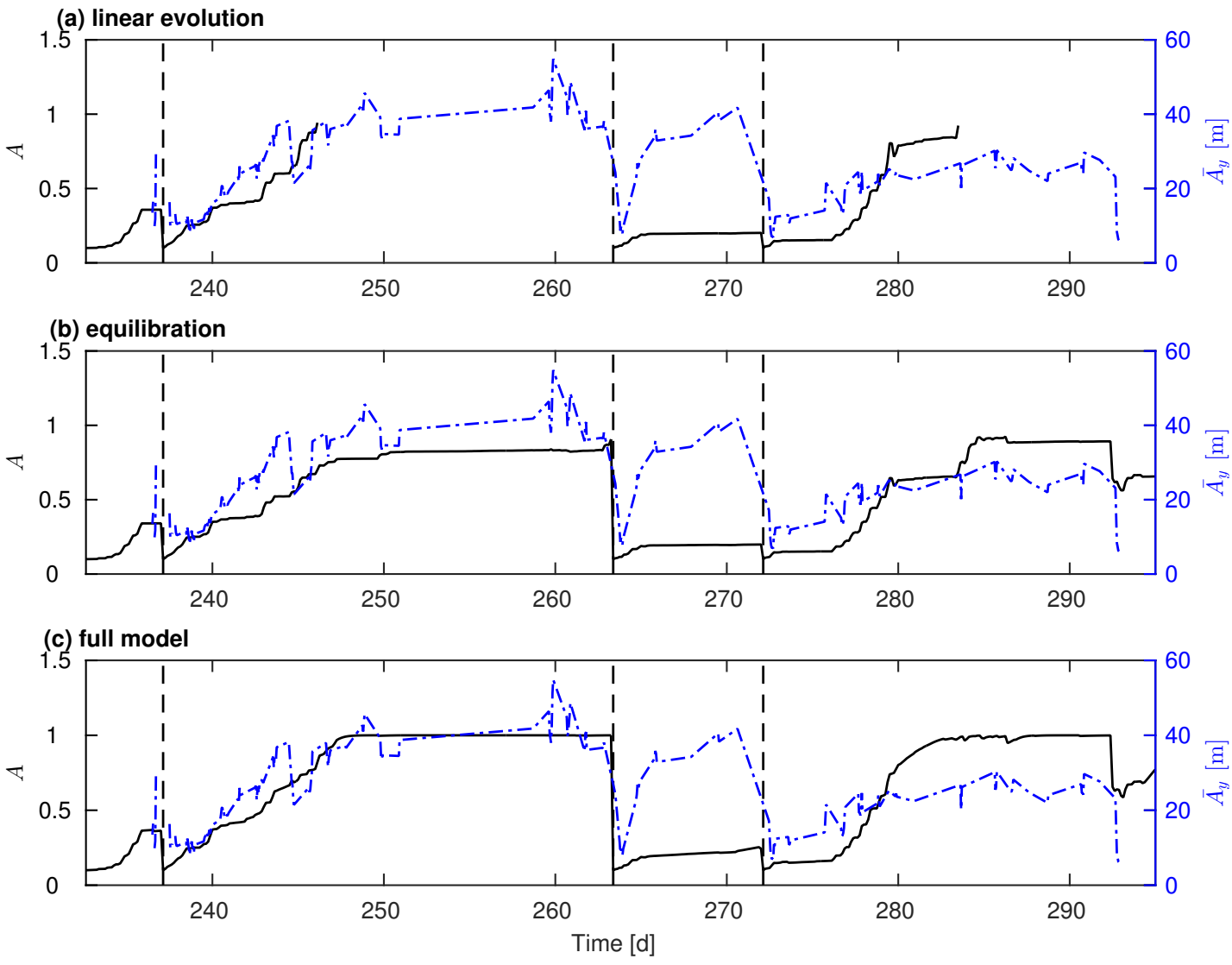

Fig. 9. Comparison between the observed and predicted dominant amplitudes, for three simulations: (a) linear evolution; (b) equilibration; (c) full model. The solid dark curve describes the amplitude of dominant lengthscale, whereas the dash-dotted blue curve refers to the observed longshore averaged horizontal amplitude $\left(\overline{A_{y}}\right)$.

\section{Discussion}

\subsection{Importance of nonlinear effects}

The most striking nonlinear effect on our simulation results is the higher harmonic interaction. A quantitative comparison between the observed and predicted lengthscales (Table 2) shows that the inclusion of higher harmonic interaction reduced the absolute and relative error of predicted and observed dominant length scale. The improvement in correspondence with the inclusion of higher harmonic interaction is also apparent in Fig. 10 where the predicted dominant lengthscale is compared to the observed lengthscale at the moments when observations could be made. The incorporation of the equilibration term is necessary. 


\begin{tabular}{l|cc} 
& Absolute error [m] & Relative error [-] \\
\hline Linear evolution & 190 & 0.54 \\
Equilibration & 168 & 0.49 \\
Full model & 108 & 0.31 \\
\hline
\end{tabular}

Table 2

The error between predicted and observed dominant length scale of the different scenarios. Note that the comparison is taken at the moments when observation could be made, and both the absolute and relative error are averaged values.
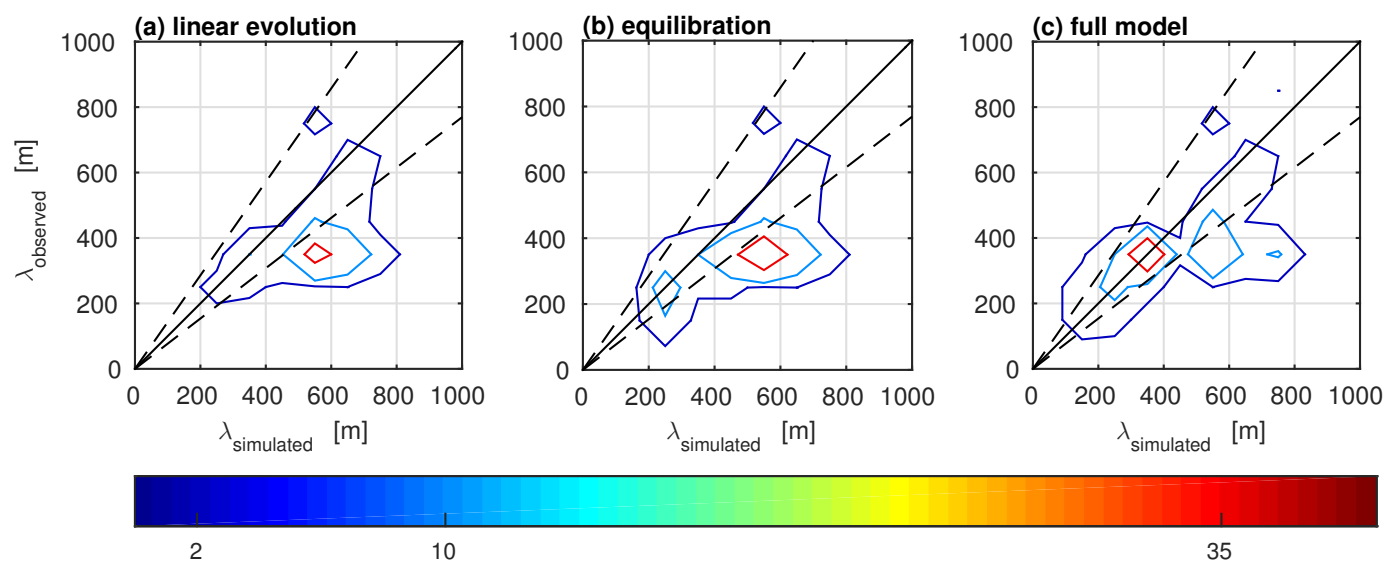

Fig. 10. Comparison between the observed and predicted dominant lengthscales, for three simulated : (a) linear evolution; (b) equilibration; (c) full model. The area between the dashed lines corresponds to relative error $<0.3$. The contour line denotes the density of data points, with red color for high density and blue color for low density, see color bar. The unit of color bar is 'number of observations (comparison) per unit area.'

\subsection{The persistence of bed pattern after storms}

In the model we have assumed that all pre-existing bed-forms have been eradicated after each storm, and the development of all lengthscales starts from the same $A_{\text {min }}$. This assumption is based on the notion that each storm is powerful enough and of long enough duration for an alongshore constant sandbar to be formed. However, field observation shows that some crescentic bed patterns can survive through a storm (Van Enckevort et al., 2004). After second storm (Fig. 6), the field observed dominant length scales stay close to the length scale before the storm, which are distinctly different to our model findings. As previously postulated in Tiessen et al. (2010), this might be due to the persistence of crescentic bed-forms throughout a comparatively less powerful storm. Moreover, apart from one observation at $\sim 700 m$ (see Fig. 6) the observed lengthscales right after the third storm stay in a narrow band close to the dominant wavelength after the second storm. This is distinctly different from the fluctuation of lengthscales observed after the first storm, and consistent 
with the aforementioned persistence of bedforms through the second storm.

To investigate this effect, we introduce a so-called persistence ratio $(\mu)$ of pre-existing bed patterns after a storm,

$$
\mu=\frac{A_{k, t_{s}^{+}}-A_{\min }}{A_{k, t_{s}^{-}}-A_{\min }},
$$

where $t_{s}^{-}\left(t_{s}^{+}\right)$refers to the time immediately before (after) the storm. The value of $\mu$ therefore ranges from 0 to 1 , where $\mu=0$ (1) means that all preexisting bed-forms have been eradicated (preserved), so the initial amplitude after storm $A_{k, t s^{+}}=A_{\min }\left(A_{k, t s^{-}}\right)$. Previously (Fig. 6) $\mu=0$ is used for all storms. Here we relate the value of $\mu$ to storm strength which is represented by the maximum wave height of each storm. From this perspective, storm 2 and 3 are of similar strength, whereas storm 1 is more powerful, see Fig. 2. We thus assume $\mu=0$ after the first, and investigate the effect of varying the (same) value of $\mu$ after second and third storms for the full model (7). In Fig. 11 (black dashed line) we see the effect of this variation in $\mu$. By allowing more bed amplitude to be preserved we observe a reduction in relative error of lengthscale as $\mu$ increases from 0 (its value in Fig. 6), and thereafter a modest increase. In fact, there is a max. error for $\mu=0$. Further research is required to clarify the mechanism lying beneath $\mu$. The sensitivity of model behaviour on $\mu$ is further discussed in $\S 5.4$.

\subsection{Energy transferred to higher harmonics}

The energy transferred from $\lambda$ to $\frac{\lambda}{2}$ is characterised by a factor $\alpha$ (see $\S 3$ ). As mentioned in $\S 3$, the value of $\alpha$ in this study was chosen based on the rate of energy transfer observed by Tiessen et al. (2011). A high value of $\alpha$ indicates a rapid transfer of energy to $\frac{\lambda}{2}$ and hence probably leads to an earlier post-storm dominance of short wavelength. It is apparent (see Fig. 11 for $\mu=0$ ) that the value used in Fig. 6 (following Tiessen et al., 2011) gives something close to the minimum relative error for the full model.

\subsection{Model sensitivity to $\mu$ and $\alpha$}

The full sensitivity of the full model behaviour to $\mu$ and $\alpha$ is shown in Fig. 11, with $0.2 \leq \alpha \leq 0.8$ and $0 \leq \mu \leq 1$ (note that we still assume that $\mu=0$ for the first, larger storm). The relative error of the predicted dominant lengthscales and observed lengthscales is smaller for non-zero $\mu$. This suggests that part of pre-existing bed pattern (and therefore lengthscale(s)) persists after second and third storms, and, by implication, that the second and third storm are not 


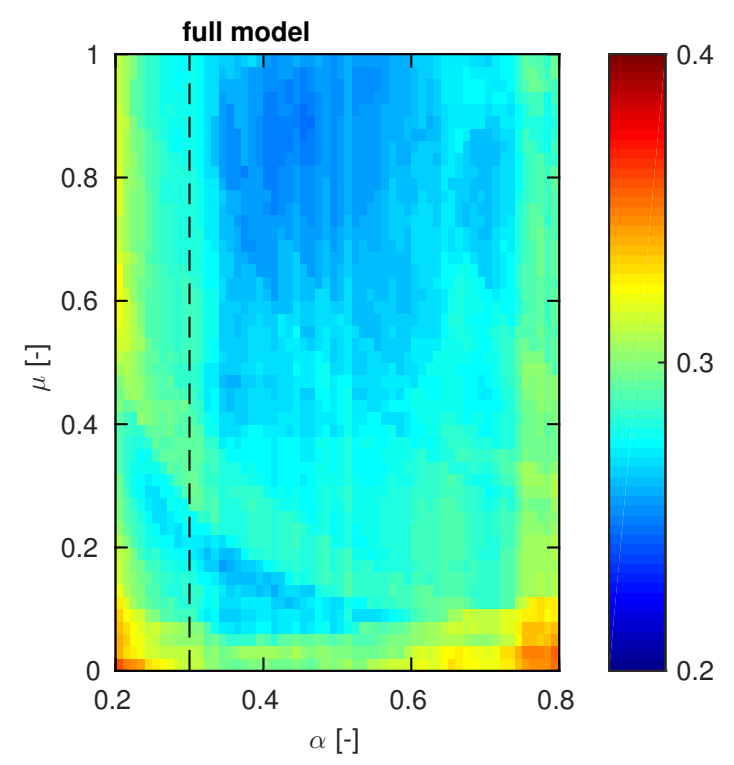

Fig. 11. sensitivity of full model behaviour on persistence ratio $\mu$ of pre-existing bed patterns and energy transfer factor $\alpha$. The vertical black dashed line refers to the choice of $\alpha=0.3$ in section 4 . Colours indicate the relative error of the predicted dominant lengthscales and observed lengthscales, with blue for low relative error and red for high relative error.

strong enough to erase all the existing bed forms. There is a region of broadly minimum error for about $0.2 \leq \mu \leq 1$ and $0.3 \leq \alpha \leq 0.6$. The conclusion appears to be that a higher $\mu$ after storm 2 and 3 leads to slightly better correspondence between prediction and observation.

The minimum error is actually achieved (Fig. 11) for $\alpha=0.41$ and $\mu=0.78$, resulting in a relative error of 0.24 (as compared to 0.31 for $\mu=0, \alpha=0.3$, see Table 2). Using these values we re-run the model for the full duration, and results are shown in Fig. 12. Additionally, we see results of the predicted dominant amplitude plotted against that observed. The predicted dominant amplitude now shows better correspondence with observation after the second storm, but poorer correspondence after the third storm. This and Fig. 10 suggest that these two storms correspond to different $\mu$ values.

\section{Conclusions}

In this study, we hypothesize that the dominant mechanisms for evolution of crescentic bar systems in nature are linear growth allied to equilibration (selflimitation) and higher harmonic generation by self-interaction. These mechanisms have been implemented into a model that would result from a weakly nonlinear perturbation analysis, but in which the coefficients of the nonlinear terms (in particular, that governing higher harmonic interactions) are set 

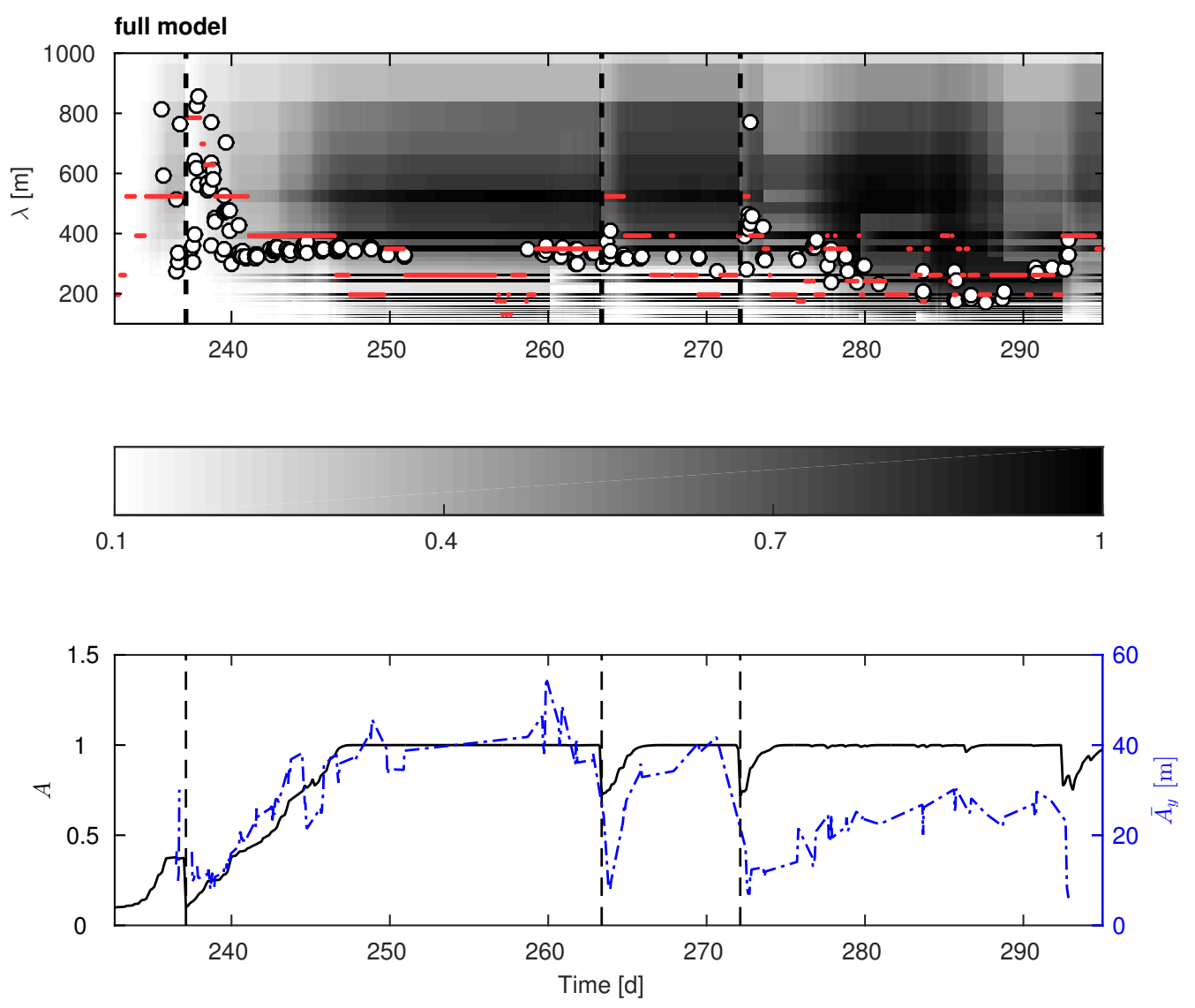

Fig. 12. Amplitude (top, figure explanation is as Fig. 6) and dominant amplitude (bottom, figure explanation similar as Fig. 9) development for the full model with $\alpha=0.41$ and $\mu=0.78$.

based on observations. This model is then used to investigate the bathymetric evolution of a crescentic-barred beach at Duck (North Carolina, USA). The model was used to reproduce a 2-month period, over which field observations were analysed by Van Enckevort et al. (2004). Results show that nonlinear effects of equilibration and higher harmonic interaction lead to significantly improved reproduction of long-term evolution of a crescentic bar system in terms of observed lengthscales.

In between storms when crescentic bars develop, their initial development corresponds well with the results from a basic linear stability analysis. The addition of a self-limitation term (Drazin and Reid, 1981) extends the predictive range of the linear stability model to the entire post-storm period. The inclusion of the term describing generation of higher-harmonics (as suggested by Tiessen et al., 2011) leads to a significant improvement in prediction of observed lengthscales. With these extra effects, an approach based on linear stability analysis can describe the observed change from immediately poststorm large lengthscales to the subsequent shorter lengthscales, related to calmer conditions in between storm events, and the subsequent stabilisation 
of the bed.

Note that the present approach is a significantly larger undertaking than that of just determining a single fastest growing mode $(F G M)$, i.e. corresponding to a single $k$ at one time, as done by Tiessen et al. (2010). Here we must determine a whole, unique growth rate curve at each time. Nonetheless, the present approach is still significantly less demanding in terms of computational time than the simulations typically required to describe the development of the whole sea bed over this area (this is generally done using a fully nonlinear model, and either $2 \mathrm{DH}$ or $3 \mathrm{D})$. An additional advantage of the currently proposed method is the significantly reduced need for beach-specific parametrisation, because detailed, spatially-variable planform-bathymetric data is not required. Similarly, only relatively idealised and schematised conditions regarding wave climate and tidal elevation are needed for a linear stability approach.

Whilst these findings represent an improvement on a linear stability model (Tiessen et al., 2010), several effects are not yet included or fully understood. For instance, the occurrence of a storm-related eradication of the crescentic bed-forms needs to be further investigated. The current research suggests that certain storms might not be strong enough to cause a wipe-out of alongshore bedforms. Additionally, the energy transferred in the higher harmonic interaction is not yet quantified. More work is needed on developing a systematic approach to deriving the amplitude equations. Doing this would allow a more complete description of long-term crescentic development (as opposed to just lengthscale and amplitude). Note also that in our approach we consider discrete wavelengths as opposed to the continuum of wavelengths that are described by a Ginsburg-Landau equation (Schielen et al., 1993). Finally, note that for some forcing conditions there is likely to be more than one physically relevant growth rate curve (see Fig. 3).

\section{Acknowledgements}

The support of the UK Engineering and Physical Sciences Research Council (EPSRC) under the MORPHINE project (grant EP/N007379/1) and of the University of Nottingham is gratefully acknowledged.

\section{References}

Calvete, D., N. Dodd, A. Falqués, and S. M. Van Leeuwen, Morphological development of rip channel systems: normal and near normal wave incidence, J. Geophys. Res., 110(C10), C10,007, doi:10.1029/2004JC002,803, 2005. 
Calvete, D., G. Coco, A. Falqués, and N. Dodd, (un)predictability in rip channel systems, Geophys. Res. Lett., 34(L05605), doi:10.1029/2006GL028,162, 2007.

Castelle, B., and B. G. Ruessink, Modeling formation and subsequent nonlinear evolution of rip channels: Timevarying versus timeinvariant wave forcing, J. Geophys. Res., 116(doi:10.1029/2011JF001997), F04,008, 2011.

Church, J. C., and E. B. Thornton, Effects of breaking of the longshore current, Coastal Eng., 20, 1-28, 1993.

Damgaard, J. S., N. Dodd, L. J. Hall, and T. J. Chesher, Morphodynamic modelling of rip channel growth, Coastal Eng., 45, 199-221, 2002.

Deigaard, R., N. Drønen, J. Fredsøe, J. H. Jensen, and M. P. Jørgensen, A morphological stability analysis for a long straight barred beach, Coastal Eng., 36(3), 171-195, 1999.

Dodd, N., P. Blondeaux, D. Calvete, H. E. de Swart, A. Falques, S. J. Hulscher, G. Rozynski, and G. Vittori, Understanding coastal morphodynamics using stability methods, Journal of coastal research, 19(4), 849-865, 2003.

Drazin, P. G., and W. H. Reid, Hydrodynamic Stability, 527 pp., Cambridge Univ. Press, New York, 1981.

Falqués, A., G. Coco, and D. A. Huntley, A mechanism for the generation of wave driven rhythmic patterns in the surf zone, J. Geophys. Res., 105(C10), 24,071-24,087, 2000.

Feddersen, F., R. T. Guza, and S. Elgar, Velocity moments in alongshore bottom stress parameterizations, J. Geophys. Res., 105(C4), 8673-8686, 2000.

Garnier, R., D. Calvete, A. Falqués, and N. Dodd, Modelling the formation and the long-term behaviour of rip channel systems from the deformation of a longshore bar, J. Geophys. Res., 113, C07,053, doi:10.1029/2007JC004,632, 2008.

Hanley, M., S. Hogart, D. Simmonds, A. Bichot, M. Colangelo, F. Bozzeda, H. Heurtefeux, B. Ondiviela, R. Ostrowski, M. Recio, R. Trude, E. Zawadzka-Kahlau, and R. Thompson, Shifting sands? coastal protection by sand banks, beaches and dunes, Coastal Engineering, 87(0), 136 $146,2014$.

Knaapen, M. A. F., and S. J. M. H. Hulscher, Use of a genetic algorithm to improve predictions of alternate bar dynamics, Water Resources Research, 39(9), 1231, 2001.

Ribas, F., A. Falqués, H. E. de Swart, N. Dodd, R. Garnier, and D. Calvete, Understanding coastal morphodynamic patterns from depth-averaged sediment concentration, Rev. Geophys., 53(2), 362-410, 2015a.

Ribas, F., A. Falqus, H. E. deSwart, N. Dodd, R. Garnier, and D. Calvete, Understanding coastal morphodynamic patterns from depth-averaged sediment concentration, Reviews of Geophysics, 53(2), 362-410, 2015b.

Schielen, R., A. Doelman, and H. de Swart, On the dynamics of free bars in straight channels, J. Fluid Mech., 252, 325-356, 1993.

Smit, M. W. J., A. J. H. M. Reniers, and M. J. F. Stive, Role of morphological variability in the evolution of nearshore sandbars, Coastal Eng., 69, 19-28, 
2012.

Soulsby, R. L., Dynamics of Marine Sands, 249 pp., Thomas Telford, London, 1997.

Tiessen, M. C. H., S. M. van Leeuwen, D. Calvete, and N. Dodd, A field test of a linear stability model for crescentic bars, Coastal Eng., 57(doi:10.1016/2009.09.002), 41-51, 2010.

Tiessen, M. C. H., N. Dodd, and R. Garnier, Development of crescentic bars for a periodically perturbed initial bathymetry, J. Geophys. Res., 116(doi:10.1029/2011JF002069), F04,016, 2011.

Van Enckevort, I. M. J., B. G. Ruessink, G. Coco, K. Suzukui, I. L. Turner, N. G. Plant, and R. A. Holman, Observations of nearshore crescentic sandbars, J. Geophys. Res., 109 (C06028), 2004.

Van Leeuwen, S. M., N. Dodd, D. Calvete, and A. Falqués, Physics of nearshore bed pattern formation under regular or random waves, J. Geophys. Res., 111 (F01023), 2006. 